\title{
Shipowner's Impact on Planned Maintenance System Database Quality Grades Resemblance Equalization
}

\section{Ladislav Stazića, Ivan Komara, Luka Mihanovićb ${ }^{\text {, Antonija }}$ Mišurac}

This paper analyzes the shipowner's role in the development process of ship's computerized planned maintenance system database, paying specific attention to his/her impact on database quality grades resemblance equalization. The paper describes the database development process from the realization that the database is needed to the installation on board vessel, and all shipowner's and developer's actions in the process.

The computerized databases of five shipping companies were tested using questionnaire developed for this purpose. The evaluation results are shown in several tables to facilitate overview and comparison of data. The paper provides the data

\section{KEY WORDS}

$\sim$ Database

$\sim$ Planned maintenance

$\sim$ Questionnaire

$\sim$ Quality evaluation of office user's workload with regard to the administration and correction of data besides database quality grades: that data serves to portrait the relation between data quality in the databases and importance given to the process of development and maintenance of the databases. The analysis of the results yields numerical values as indicators of shipowner's input on the resemblance of grades of both scenarios and on the database quality grades resemblance equalization.

The conclusion shows how the shipowner affects the ship's computerized planned maintenance system database and what actions should be performed to obtain the final product (database, i.e. data in the database) as good as possible and, thus, maintenance of the ship and the whole system.

The expected results of the paper are:

- $\quad$ To analyze in detail the database ordering and development process,

\footnotetext{
- To pinpoint controlling areas for the database quality,

To analyze the shipowner's impact on the database quality grades resemblance equalization.
}

\section{INTRODUCTION}

The database developer (database development team) and the shipowner are two major factors that influence ship's computerized planned maintenance system database quality. The database developer's interests in the process are to fulfill the shipowner's requests as soon as possible with the costs as low as possible. During the database development, i.e. data entry process, deficiencies in the database may appear (Wang 
and Strong, 1996), (Rabin, 2002). The deficiencies depend upon the database development team, their expertise, knowledge, abilities, etc. As the database development team will modify the data entry and database development according to their characteristics and abilities, the deficiencies created by the team will be present in all their databases. The quality evaluation grades of the databases made by one development team will therefore have quite a great resemblance which will tend toward equivalence (resemblance of $100 \%$ ).

The shipowner's interests in the database development process are to procure a database as good as possible, made according to his needs and requirements, with the price as low as possible. The shipowner influences the database quality by establishing a precise list of database requirements and by measuring the quality of data entered. With these actions he provides a certain amount of uniformity of company databases. The measurement of data quality "helps during the course of a project, to assess its progress, to take corrective action based on this assessment, and to evaluate the impact of such action" (Basili et al., 1994).

Testing of the evaluation methodology for ship's Planned Maintenance System database was performed on five databases and their resemblance was analyzed (Table 1).

Table 1.

Resemblance of grades of Methodology testing (in \%).

\begin{tabular}{llllll} 
& Database 1 & Database 2 & Database 3 & Database 4 & Database 5 \\
\hline Database 1 & 100 & 98.33 & 96.66 & 97.50 & 90.00 \\
\hline Database 2 & 98.33 & 100 & 95.00 & 95.83 & 90.83 \\
\hline Database 3 & 96.66 & 95.00 & 100 & 99.17 & 86.66 \\
\hline Database 4 & 97.50 & 95.83 & 99.17 & 100 & 87.50 \\
\hline Database 5 & 90.00 & 90.83 & 86.66 & 87.50 & 100
\end{tabular}

The evaluated databases had resemblance of quality grades varying from 95 to $100 \%$ when compared within one company (databases 1 to 4 ) and 86.66 to $90.83 \%$ when compared with a database of a different provider (database 5). The resemblance difference of only $5 \%$ during the comparison of grades of two different shipowners was unexpectedly low and created several questions: whether the results were interpreted wrongly, whether the results were accidental due to a small sample, are the results such because of the inactivity of the shipowner, or something else. The evaluated databases, besides a high resemblance of grades, had similar characteristics and deficiencies. That was pointing towards a lack of the established data quality measurement and supervision system for the database and, therefore, a flaw made by the shipowner. The reason for the shipowner's flaw can be traced to the resistance towards changes in the work process, not knowing the system, and accidental mistakes. Introducing new systems (costs) in the maintenance process without creating new values can lead to resistances in the system (Gackowski, 2006), especially in the shipping industry which shows a high level of conservatism (Manuel, 2012). Identifying place and the moment of creation of data quality problems will direct the search focus to solving these problems (Strong et al., 1997).

The sample size during testing of evaluation methodology was small, nobody "gambles his research hypotheses on small samples without realizing that the odds against him are unreasonably high" (Tversky and Kahneman, 1971). New, larger research was performed with the intention to establish why small sample results were unexpected and how evaluation grades will look on the larger sample. New research had the primary task to determine where and how the data in databases became deficient and why the same or similar deficiencies appear for different companies in the database development process.

Chapter 2 presents the analysis of the development process of ship's computerized planned maintenance system database together with the sequence diagram of the shipowner's (and developer's) actions which influence database quality and which are shown in the diagram.

The overview of the research starts with the results of database evaluation of five shipping companies. The databases for ship's computerized planned maintenance system were evaluated using the dedicated questionnaire developed for the purpose (Table 2). The research is divided into two scenarios shown in separate chapters.

Chapter 3 presents the evaluation grades according to the first scenario. The scenario replicates the conditions of research during Evaluation methodology testing (Table 1) where all the databases were built by the same development team. Sixteen databases from three companies were evaluated during this stage of research. The research according to the first scenario had the purpose to establish how the shipowner's actions affect 
creation of database specific features and database quality grades resemblance equalization.

The second scenario covers two companies developing their databases by different development teams. The scenario is described in Chapter 4; twenty-eight databases were evaluated during this phase of the research. The research according to the second scenario had the purpose to establish how the shipowner's actions affect equalizing of quality grades of databases which were very different initially.

Insight into the number of office users and their workload with regard to administration and correction of data in the database is also shown in the paper to enable an additional shipowner's influence.

An analysis of the data in discussion was performed by calculation of the average grade value, calculation and analysis of the resemblance of databases and comparison of the noted major deficiencies. The results are analyzed and compared on company levels and all together, among all the companies.
The conclusion of the paper gives the answer how shipowner's actions influence data quality in the database and explains the results of the research during Evaluation methodology testing.

As shipping companies allowed access to their databases and real data strictly under no disclosure condition, in the paper they will be named as companies A, B, C, D, E.

\section{DATABASE DEVELOPMENT PROCESS}

Database development process (Beynon-Davies, 2004), (Watt and Eng, 2014) is modified and applied for ship planned maintenance (Figure 1). The whole process starts with the decision of the shipowner that it is necessary to order creation of ship's Planned Maintenance System (PMS) database, followed with determination of requirements which the database must fulfill.

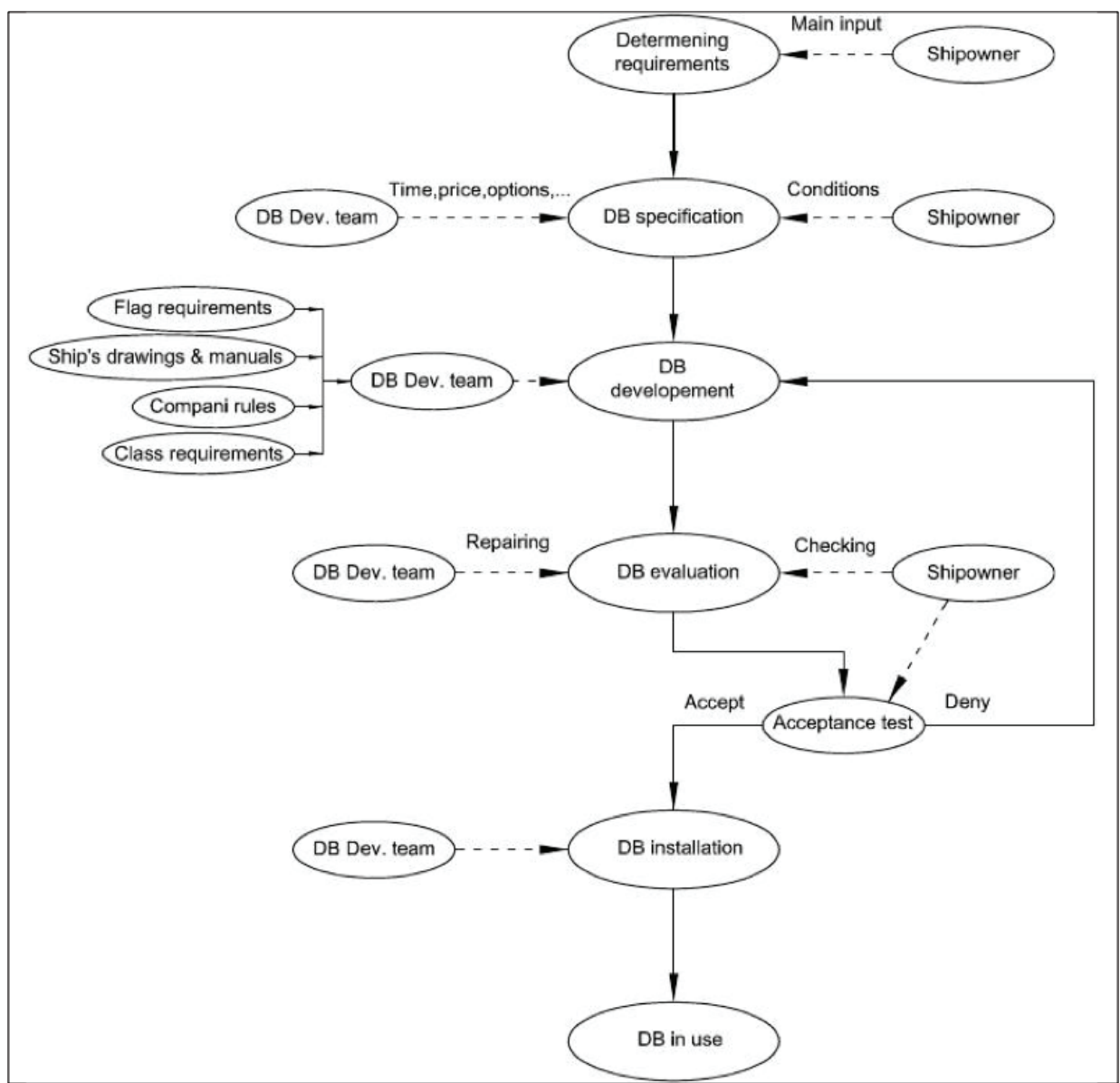

Figure 1.

PMS DB (Planned Maintenance System Database) development process sequence diagram (based on Watt and Eng, 2014). 
The determination of requirements is performed by the shipowner and can be performed with four methods: asking, deriving from an existing information system, synthesis from characteristics of the utilized system, discovering from experimentation with an evolving information system application (Davis, 1982), (Davis and Olson, 1985).

Contracting of the database is the second important step in the database development process and it is performed by both sides, the shipowner and the developer. During the negotiations, they must agree on quite a number of details such as DB data content, price, development time, date of delivery (installation), modes of control, modes of delivery, etc. The shipowner and the developer must specify in detail all the sources of information to be included in DB to avoid receiving of inadequate final product (bad databases).

In the next phase, the shipowner must take care to deliver to the development team complete documentation needed for the insertion of data into DB, consisting of: maker's Instruction manuals for the ship's equipment; the shipowner's company policies and rules; the classification society requirements for the vessel, for example American Bureau of Shipping (ABS rules for conditions of classification, 2016), Det Norske Veritas Germanischer Lloyd (DNV rules for classification of ships, 2003), or Croatian Register of Shipping (CRS rules for classification of ships, 2014); ship's flag state requirements (Guidelines for flag State inspections under the Maritime Labour Convention, 2006).

As data insertion is performed manually, it is susceptible to a significant amount of errors. The most common source of data inaccuracy is the person entering the data and that will always remain a significant problem of data entry (Maydanchik, 2007). After DB developer finishes insertion of data into the database, the shipowner should organize the inspection of data in DB and discover mistakes and deficiencies which should be rectified before the final DB acceptance test.

The final or acceptance test is a test arranged by the shipowner to verify if the whole process of the development was complete and correct (Rogers, 2004). That is a crucial action for the success of the whole project (Miller and Collins, 2001), in case of a positive assessment, DB is installed on board while in case of a negative assessment $D B$ is returned to the developer to rectify the deficiencies.

\section{RESULTS OF THE FIRST QUALITY EVALUATION OF DATABASES}

As the law of large numbers guarantees that very large samples will indeed be highly representative (Tversky and
Kahneman, 1971), quality evaluation of more databases was arranged. The evaluation was performed using the questionnaire specially developed for the purpose (Table 2).

The evaluation grades of three shipping companies are linked together for an easier overview (Table 3 ). The evaluations of databases A-1, A-2, A-3, C-1, C-2, C-3, C-4, C-5, C-6, C-7, C-8 were performed by employees of the shipping companies familiar with the computerized PMS and ship's maintenance, therefore the condition of knowing DB structure and data as well as the company rules with regard to ship's maintenance was fulfilled. Analysis of databases B-1, B-2, B-3, B-4, B-5 was performed by one of authors of the paper. Since the evaluator was not familiar with the company rules, evaluation grades might have certain amount of subjectivity.

As an addition to this research there is data about the companies $A, B$, and $C$ office user's workload with regard to the administration and correction of data (Table 4), given in average daily manhours per DB.

\section{RESULTS OF THE SECOND QUALITY EVALUATION OF DATABASES}

The second scenario follows the database quality evaluation grades when the shipowner is the same and the database development team different. The databases of two shipping companies, D and E, were evaluated according to this scenario. The company D's database evaluation (Table 5) was performed by their PMS administrator.

In cooperation with office users, data were collected about the company D's office user's workload with regard to the administration and correction of databases (Table 6), given in average daily manhours per database.

The company E's database evaluation (Table 7) was performed by their junior superintendent who is involved in the maintenance of vessels and works with computerized PMS on daily basis.

The company E's office user's workload with regard to the administration and correction of databases (Table 8) was also taken into consideration; it is presented in the average daily manhours per database. 
Table 2.

Computerized PMS database evaluation questionnaire.

Area

Machinery and equipment

\section{Jobs inside DB}

Special jobs and rules - DB jobs general

Spare parts

Miscellaneous

\section{Question}

Grade

\begin{tabular}{|l|}
\hline 01. \\
\hline 02. \\
\hline 03. \\
\hline
\end{tabular}

Is all the machinery and equipment included in the database?

Is all the included equipment marked properly and uniquely, according to their shipboard location and markings?

Is all the necessary machinery divided to subcomponents (to smaller subsystems) in a logical manner?

04. Does the machinery or equipment have a larger number of subcomponents than necessary?

05. Is there equipment or machinery listed in the database more than once, or do they have the same markings or names?

06. Is the data about the manufacturer, type and serial number entered to all the relevant items?

07. Do all the equipment and machinery entries have the same style, abbreviations, and markings?

08. Do all the devices in the DB have linked maintenance plan according to the manufacturer's recommendation?

09. Are the manufacturer's recommendations grouped according to devices, periods and company maintenance rules?

10. Are all the jobs required by the company policy included in the DB? (e.g. SMS - Safety Management System)?

11. Are all the jobs based on the manufacturer's recommendation changed due to the company policy (if exists)?

12. Are all the jobs required by flag state rules and regulations included in the DB?

Are all the jobs required by class society included in the DB?

14. Is there a number of smaller jobs which can be grouped together?

15. Is fire detection sensor list inserted into the DB together with the testing plan?

16. Is the alarm system and its testing program entered in the DB?

17. Is PMS self-improvement program inserted into the DB, and is there control mechanism for PMS DB self-improvement program?

18. Is the critical equipment marked according to company SMS?

19. Are job descriptions written clearly and straightforward?

20. Are jobs created and grouped according to multiplier principle?

21. Are all the same type jobs coming from different sources synchronized?

22. Are all the same jobs resulting from different requirements (sources) merged?

23. Are all the required spare parts included in the database?

24. Are spare parts distributed to proper equipment and machinery?

25. Are all the spare parts properly marked; do they have sufficient data for ordering?

26. Is the company critical spare parts list inserted in the DB?

27. Do all the spare parts have the same style, abbreviations, markings, etc.?

28. Are there spare parts entered several times?

29. Are all the users inserted in the $\mathrm{DB}$, and are all the access rights defined in order?

30. Is there any other deficiency noted in the computerized PMS database? 
Table 3.

Results of the first quality evaluation of databases.

\section{Question}

\section{Database}

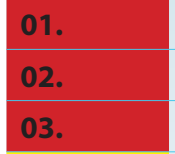

\section{$\begin{array}{lllllllll}\text { A-1 } & \text { A-2 } & \text { A-3 } & \text { B-1 } & \text { B-2 } & \text { B-3 } & \text { B-4 } & \text { B-5 } & \text { C-1 }\end{array}$}

04.

05.

$\begin{array}{lllllll}5 & 3 & 5 & 5 & 4 & 5 & 5\end{array}$
$\begin{array}{lllllllllllllll}3 & 5 & 5 & 4 & 5 & 5 & 4 & 5 & 5 & 4 & 5 & 5 & 5 & 4 & 5\end{array}$

C-2 C-3

$\begin{array}{lllll}C-4 & \text { C-5 } & \text { C-6 } & \text { C-7 } & \text { C-8 }\end{array}$

06. $\begin{array}{llllllllll}3 & 4 & 5 & 5 & 5 & 5 & 5 & 4 & 4 & 5\end{array}$

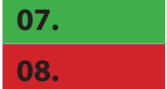

09.

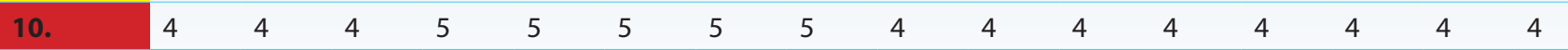

11. $5 \begin{array}{llllllllllllllll} & 5 & 5 & 5 & 4 & 4 & 4 & 4 & 4 & 2 & 3 & 2 & 2 & 3 & 2 & 1\end{array}$

\begin{tabular}{lllllllllllllllll} 
12. & 4 & 4 & 4 & 5 & 5 & 5 & 5 & 5 & 3 & 3 & 3 & 3 & 3 & 3 & 3 & 3 \\
\hline 13. & 5 & 5 & 5 & 5 & 5 & 5 & 5 & 5 & 5 & 5 & 5 & 5 & 5 & 5 & 5 & 5 \\
\hline 14. & 4 & 4 & 4 & 5 & 5 & 5 & 5 & 5 & 4 & 4 & 4 & 4 & 4 & 4 & 4 & 4 \\
\hline 15. & 5 & 1 & 3 & 5 & 5 & 5 & 5 & 5 & 5 & 5 & 5 & 1 & 5 & 5 & 1 & 5 \\
\hline 16. & 5 & 1 & 2 & 5 & 5 & 5 & 5 & 5 & 5 & 5 & 5 & 3 & 5 & 5 & 1 & 5 \\
\hline 17. & 5 & 5 & 5 & 5 & 5 & 5 & 5 & 5 & 5 & 5 & 5 & 5 & 5 & 5 & 5 & 5 \\
\hline 18. & 5 & 5 & 5 & 5 & 5 & 5 & 5 & 5 & 5 & 5 & 5 & 5 & 5 & 5 & 5 & 5 \\
\hline 19. & 5 & 5 & 5 & 5 & 5 & 5 & 5 & 5 & 4 & 4 & 4 & 4 & 4 & 4 & 4 & 4 \\
\hline 20. & 4 & 4 & 4 & 3 & 3 & 3 & 3 & 3 & 4 & 4 & 4 & 4 & 4 & 4 & 4 & 4 \\
\hline 21. & 5 & 5 & 5 & 5 & 5 & 5 & 5 & 5 & 4 & 4 & 4 & 4 & 4 & 4 & 4 & 4 \\
22. & 5 & 5 & 5 & 5 & 5 & 5 & 5 & 5 & 4 & 4 & 4 & 4 & 4 & 4 & 4 & 4 \\
\hline 23. & 5 & 2 & 4 & 5 & 5 & 5 & 5 & 5 & 4 & 4 & 4 & 3 & 4 & 4 & 3 & 4 \\
\hline 24. & 5 & 5 & 5 & 5 & 5 & 5 & 5 & 5 & 5 & 5 & 5 & 5 & 5 & 5 & 5 & 5 \\
\hline 25. & 5 & 2 & 4 & 5 & 5 & 5 & 5 & 5 & 4 & 4 & 4 & 4 & 4 & 4 & 4 & 4 \\
\hline 26. & 4 & 4 & 4 & 3 & 3 & 3 & 3 & 3 & 5 & 5 & 5 & 5 & 5 & 5 & 5 & 5 \\
\hline 27. & 5 & 2 & 4 & 5 & 5 & 5 & 5 & 5 & 5 & 5 & 5 & 5 & 5 & 5 & 5 & 5 \\
\hline 28. & 4 & 4 & 4 & 5 & 5 & 5 & 5 & 5 & 5 & 5 & 5 & 5 & 5 & 5 & 5 & 5 \\
\hline 29. & 5 & 5 & 5 & 5 & 5 & 5 & 5 & 5 & 5 & 5 & 5 & 5 & 5 & 5 & 5 & 5 \\
\hline 30. & 5 & 5 & 5 & 5 & 5 & 5 & 5 & 5 & 4 & 4 & 4 & 3 & 4 & 4 & 3 & 4
\end{tabular}

Table 4.

Office user's workload with regard to administration and correction of the data.

$\begin{array}{lllllllllllllllll}\text { Database } & \text { A-1 } & \text { A-2 } & \text { A-3 } & \text { B-1 } & \text { B-2 } & \text { B-3 } & \text { B-4 } & \text { B-5 } & \text { C-1 } & \text { C-2 } & \text { C-3 } & \text { C-4 } & \text { C-5 } & \text { C-6 } & \text { C-7 } & \text { C-8 } \\ \text { Work hrs. } & 0.3 & 0.3 & 0.3 & 1.5 & 1.5 & 1.5 & 1.5 & 1.5 & 0.8 & 0.8 & 0.8 & 0.8 & 0.8 & 0.8 & 0.8 & 0.8\end{array}$

Remarks: Data were collected in cooperation with PMS DB administrators in the shipping companies. The number under Work hours represents average daily manhours used per administration and correction of each database. 
Table 5.

Company D's database evaluation grades.

\begin{tabular}{|c|c|c|c|c|c|c|c|c|c|c|c|c|c|c|c|c|c|c|c|}
\hline \multirow{2}{*}{ Question } & \multicolumn{19}{|c|}{ Database } \\
\hline & D-1 & D-2 & D-3 & D-4 & D-5 & D-6 & D-7 & D-8 & D-9 & D-10 & D-11 & D-12 & D-13 & D-14 & D-15 & D-16 & D-17 & D-18 & D-19 \\
\hline 01. & 3 & 4 & 4 & 4 & 4 & 5 & 5 & 4 & 3 & 4 & 4 & 4 & 5 & 4 & 4 & 3 & 4 & 4 & 4 \\
\hline 02. & 3 & 5 & 5 & 5 & 4 & 5 & 5 & 4 & 3 & 5 & 5 & 5 & 5 & 5 & 5 & 3 & 4 & 5 & 5 \\
\hline 03. & 2 & 4 & 4 & 3 & 4 & 5 & 5 & 4 & 5 & 5 & 4 & 5 & 5 & 5 & 5 & 4 & 4 & 4 & 4 \\
\hline 04. & 5 & 4 & 4 & 4 & 2 & 5 & 5 & 4 & 5 & 5 & 4 & 5 & 4 & 4 & 4 & 5 & 2 & 4 & 4 \\
\hline 05. & 5 & 5 & 5 & 5 & 5 & 5 & 5 & 5 & 5 & 5 & 5 & 5 & 5 & 5 & 5 & 5 & 5 & 5 & 5 \\
\hline 06. & 2 & 4 & 4 & 3 & 4 & 5 & 5 & 4 & 3 & 4 & 4 & 5 & 4 & 5 & 5 & 3 & 4 & 4 & 4 \\
\hline 07. & 5 & 5 & 5 & 5 & 5 & 5 & 5 & 5 & 5 & 5 & 5 & 5 & 5 & 5 & 5 & 5 & 5 & 5 & 5 \\
\hline 08. & 3 & 5 & 4 & 4 & 3 & 5 & 5 & 5 & 2 & 5 & 5 & 5 & 4 & 5 & 5 & 2 & 4 & 4 & 3 \\
\hline 09. & 3 & 5 & 4 & 4 & 4 & 4 & 4 & 4 & 2 & 4 & 5 & 5 & 4 & 4 & 4 & 3 & 4 & 4 & 4 \\
\hline 10. & 1 & 2 & 2 & 2 & 2 & 2 & 2 & 2 & 1 & 2 & 1 & 2 & 1 & 2 & 2 & 1 & 2 & 2 & 2 \\
\hline 11. & 1 & 1 & 1 & 1 & 1 & 1 & 1 & 1 & 1 & 1 & 1 & 1 & 1 & 1 & 1 & 1 & 1 & 1 & 1 \\
\hline 12. & 1 & 1 & 1 & 1 & 1 & 1 & 1 & 1 & 1 & 1 & 1 & 1 & 1 & 1 & 1 & 1 & 1 & 1 & 1 \\
\hline 13. & 1 & 1 & 1 & 1 & 1 & 1 & 4 & 1 & 1 & 4 & 1 & 1 & 1 & 1 & 1 & 1 & 1 & 4 & 1 \\
\hline 14. & 1 & 1 & 4 & 1 & 1 & 1 & 4 & 2 & 2 & 4 & 1 & 1 & 1 & 3 & 1 & 1 & 1 & 4 & 2 \\
\hline 15. & 2 & 1 & 4 & 1 & 2 & 1 & 4 & 3 & 3 & 4 & 1 & 1 & 1 & 4 & 1 & 2 & 1 & 5 & 3 \\
\hline 16. & 1 & 1 & 3 & 1 & 1 & 1 & 3 & 1 & 2 & 3 & 1 & 1 & 2 & 3 & 1 & 2 & 1 & 3 & 2 \\
\hline 17. & 1 & 1 & 1 & 1 & 1 & 1 & 1 & 1 & 1 & 1 & 1 & 1 & 1 & 1 & 1 & 1 & 1 & 1 & 1 \\
\hline 18. & 1 & 1 & 1 & 1 & 1 & 1 & 1 & 1 & 1 & 1 & 1 & 1 & 1 & 1 & 1 & 1 & 1 & 1 & 1 \\
\hline 19. & 4 & 4 & 5 & 4 & 4 & 5 & 5 & 4 & 4 & 5 & 5 & 5 & 5 & 5 & 4 & 4 & 4 & 5 & 4 \\
\hline 20. & 2 & 5 & 5 & 3 & 4 & 5 & 5 & 4 & 2 & 4 & 4 & 4 & 5 & 4 & 5 & 2 & 3 & 5 & 4 \\
\hline 21. & 2 & 4 & 4 & 3 & 4 & 5 & 5 & 4 & 3 & 4 & 4 & 4 & 5 & 4 & 4 & 3 & 4 & 5 & 4 \\
\hline 22. & 5 & 5 & 5 & 5 & 5 & 5 & 5 & 5 & 5 & 5 & 5 & 5 & 5 & 5 & 5 & 5 & 5 & 5 & 5 \\
\hline 23. & 1 & 4 & 5 & 2 & 4 & 4 & 2 & 5 & 1 & 4 & 2 & 4 & 5 & 4 & 4 & 1 & 4 & 3 & 3 \\
\hline 24. & 1 & 4 & 5 & 4 & 4 & 4 & 4 & 5 & 1 & 5 & 4 & 4 & 5 & 4 & 4 & 1 & 4 & 4 & 3 \\
\hline 25. & 1 & 4 & 4 & 3 & 3 & 4 & 4 & 5 & 1 & 4 & 5 & 4 & 5 & 4 & 4 & 1 & 4 & 4 & 4 \\
\hline 26. & 1 & 1 & 1 & 1 & 1 & 1 & 1 & 1 & 1 & 1 & 1 & 1 & 1 & 1 & 1 & 1 & 1 & 1 & 1 \\
\hline 27. & 5 & 5 & 5 & 5 & 5 & 5 & 5 & 5 & 5 & 5 & 5 & 5 & 5 & 5 & 5 & 5 & 5 & 5 & 5 \\
\hline 28. & 5 & 4 & 4 & 5 & 3 & 4 & 4 & 5 & 4 & 5 & 5 & 5 & 4 & 5 & 5 & 5 & 5 & 5 & 5 \\
\hline 29. & 5 & 5 & 5 & 5 & 5 & 5 & 5 & 5 & 5 & 5 & 5 & 5 & 5 & 5 & 5 & 5 & 5 & 5 & 5 \\
\hline 30. & 1 & 5 & 5 & 5 & 1 & 5 & 5 & 5 & 1 & 5 & 5 & 3 & 5 & 5 & 5 & 1 & 5 & 3 & 1 \\
\hline
\end{tabular}

\section{Table 6.}

Company D's office user's workload with regard to administration and correction of the database.

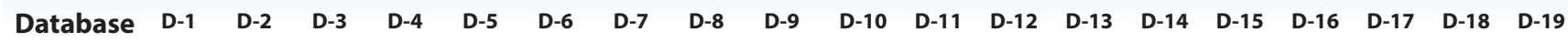
$\begin{array}{llllllllllllllllllll}\text { Work hrs. } & 0.06 & 0.06 & 0.06 & 0.06 & 0.06 & 0.06 & 0.06 & 0.06 & 0.06 & 0.06 & 0.06 & 0.06 & 0.06 & 0.06 & 0.06 & 0.06 & 0.06 & 0.06 & 0.06\end{array}$ Remark: The number under Work hours represents the average daily manhours used per administration and correction of each database. 
Table 7.

Company E's database evaluation grades.

\begin{tabular}{|c|c|c|c|c|c|c|c|c|c|}
\hline \multirow{2}{*}{ Question } & \multicolumn{9}{|c|}{ Database } \\
\hline & E-1 & $E-2$ & $E-3$ & $E-4$ & E-5 & $E-6$ & E-7 & $E-8$ & E-9 \\
\hline 01. & 5 & 5 & 5 & 5 & 5 & 5 & 5 & 5 & 5 \\
\hline 02. & 5 & 5 & 5 & 5 & 5 & 5 & 5 & 5 & 5 \\
\hline 03. & 5 & 5 & 5 & 5 & 5 & 5 & 5 & 5 & 5 \\
\hline 04. & 5 & 5 & 5 & 5 & 5 & 5 & 5 & 5 & 5 \\
\hline 05. & 5 & 5 & 5 & 5 & 5 & 5 & 5 & 5 & 5 \\
\hline 06. & 4 & 4 & 4 & 4 & 4 & 4 & 4 & 4 & 4 \\
\hline 07. & 4 & 4 & 4 & 4 & 4 & 4 & 4 & 4 & 4 \\
\hline 08. & 5 & 5 & 5 & 5 & 5 & 5 & 5 & 5 & 5 \\
\hline 09. & 4 & 4 & 4 & 4 & 4 & 4 & 4 & 4 & 4 \\
\hline 10. & 5 & 5 & 5 & 5 & 5 & 5 & 5 & 5 & 5 \\
\hline 11. & 4 & 4 & 4 & 4 & 4 & 4 & 4 & 4 & 4 \\
\hline 12. & 4 & 4 & 4 & 4 & 4 & 4 & 4 & 4 & 4 \\
\hline 13. & 5 & 5 & 5 & 5 & 5 & 5 & 5 & 5 & 5 \\
\hline 14. & 1 & 1 & 1 & 1 & 1 & 1 & 1 & 1 & 1 \\
\hline 15. & 5 & 5 & 5 & 5 & 5 & 5 & 5 & 5 & 5 \\
\hline 16. & 5 & 5 & 5 & 4 & 4 & 5 & 5 & 4 & 4 \\
\hline 17. & 5 & 5 & 5 & 5 & 5 & 5 & 5 & 5 & 5 \\
\hline 18. & 5 & 5 & 5 & 5 & 5 & 5 & 5 & 5 & 5 \\
\hline 19. & 5 & 5 & 5 & 5 & 5 & 5 & 5 & 5 & 5 \\
\hline 20. & 5 & 5 & 5 & 5 & 5 & 5 & 5 & 5 & 5 \\
\hline 21. & 4 & 4 & 4 & 4 & 4 & 4 & 4 & 4 & 4 \\
\hline 22. & 5 & 5 & 5 & 5 & 5 & 5 & 5 & 5 & 5 \\
\hline 23. & 4 & 5 & 5 & 5 & 4 & 5 & 5 & 4 & 4 \\
\hline 24. & 5 & 5 & 5 & 5 & 5 & 5 & 5 & 5 & 5 \\
\hline 25. & 4 & 4 & 4 & 4 & 4 & 4 & 4 & 4 & 4 \\
\hline 26. & 5 & 5 & 5 & 5 & 5 & 5 & 5 & 5 & 5 \\
\hline 27. & 5 & 5 & 5 & 5 & 5 & 5 & 5 & 5 & 5 \\
\hline 28. & 4 & 4 & 4 & 4 & 4 & 4 & 4 & 4 & 4 \\
\hline 29. & 5 & 5 & 5 & 5 & 5 & 5 & 5 & 5 & 5 \\
\hline 30. & 5 & 5 & 5 & 5 & 5 & 5 & 5 & 5 & 5 \\
\hline
\end{tabular}

Table 8.

Company E's office user's workload with regard to the administration and correction of database.

$\begin{array}{llllllllll}\text { Database } & E-1 & \text { E-2 } & \text { E-3 } & \text { E-4 } & \text { E-5 } & \text { E-6 } & \text { E-7 } & \text { E-8 } & \text { E-9 }\end{array}$

$\begin{array}{llllllllll}\text { Work hrs. } & 1 & 1 & 1 & 1 & 1 & 1 & 1 & 1 & 1\end{array}$

Remark: The number under Work hours represents the average daily manhours used per administration and correction of each database. 


\section{ANALYSIS OF DATABASE EVALUATION RESULTS}

Analysis of the database evaluation results is a step that follows the process of evaluation. The analysis started by the calculation of summation of grades and the average grade value for all the databases. The results are shown separately for the first quality evaluation (Table 9) and the second quality evaluation (Table 10 and Table 11).

Table 9.

Summation of grades and the average grade value for the first quality evaluation.

\begin{tabular}{llllllllllllllllll} 
Database & A-1 & A-2 & A-3 & B-1 & B-2 & B-3 & B-4 & B-5 & C-1 & C-2 & C-3 & C-4 & C-5 & C-6 & C-7 & C-8 \\
\hline $\begin{array}{l}\text { Sum. of } \\
\text { grades }\end{array}$ & 142 & 119 & 133 & 143 & 142 & 144 & 143 & 143 & 131 & 131 & 131 & 124 & 132 & 131 & 118 & 134 \\
\hline $\begin{array}{l}\text { Av. grade } \\
\text { value }\end{array}$ & 4.733 & 3.967 & 4.433 & 4.767 & 4.733 & 4.800 & 4.767 & 4.767 & 4.367 & 4.367 & 4.367 & 4.133 & 4.400 & 4.367 & 3.933 & 4.467 \\
\end{tabular}

Table 10.

Summation of grades and the average grade value for company D.

\begin{tabular}{|c|c|c|c|c|c|c|c|c|c|c|c|c|c|c|c|c|c|c|c|}
\hline Database & D-1 & D-2 & D-3 & D-4 & D-5 & D-6 & D-7 & D-8 & D-9 & D-10 & D-11 & D-12 & D-13 & D-14 & D-15 & D-16 & D-17 & D-18 & D-19 \\
\hline $\begin{array}{l}\text { Sum. of } \\
\text { grades }\end{array}$ & 74 & 101 & 110 & 92 & 89 & 106 & 115 & 105 & 79 & 115 & 100 & 103 & 106 & 110 & 103 & 78 & 95 & 111 & 96 \\
\hline $\begin{array}{l}\text { Av. grade } \\
\text { value }\end{array}$ & 2.467 & 3.367 & 3.667 & 3.067 & 2.967 & 3.533 & 3.833 & 3.500 & 2.633 & 3.833 & 3.333 & 3.433 & 3.533 & 3.667 & 3.433 & 2.600 & 3.167 & 3.700 & 3.200 \\
\hline
\end{tabular}

Table 11.

Summation of grades and the average grade value for company $E$.

\begin{tabular}{llllllllll} 
Database & E-1 & E-2 & E-3 & E-4 & E-5 & E-6 & E-7 & E-8 & E-9 \\
\hline $\begin{array}{l}\text { Sum. of } \\
\text { grades }\end{array}$ & 137 & 138 & 138 & 137 & 136 & 138 & 138 & 136 & 136 \\
\hline $\begin{array}{l}\text { Av. grade } \\
\text { value }\end{array}$ & 4.567 & 4.600 & 4.600 & 4.567 & 4.533 & 4.600 & 4.600 & 4.533 & 4.533 \\
\hline
\end{tabular}

The resemblance analysis of the evaluation grades was made according to Equation 1 (Stazić at al, 2017) as the next step of the analysis:

$S=100-\frac{100}{n Q} \Sigma\left|R i_{b j}-R i_{b k}\right| \cdot \frac{1}{n G-1}[\%]$

where:

$S$ - resemblance of grades of two databases

$\mathrm{nQ}$ - total number of questions

$\mathrm{Ri}$-answer on $i^{\text {th }}$ question $(\mathrm{i}=1,2 \ldots \mathrm{n})$

bj $-j^{\text {th }}$ evaluated database,

bk $-k^{\text {th }}$ evaluated database; $(j, k=1,2 \ldots m, j \neq k)$

$\mathrm{nG}$ - total number of grades
The calculated results are divided into three tables, one for the first quality evaluation (Table 12), and the other two for the second quality evaluation (Table 13 and Table 14).

The final part of the analysis of quality evaluation results is made with an overview of the questions which received grades 1, 2 or 3 (i.e. received lower grades). Different characteristics of databases are noted during this part of analysis. Company $\mathrm{E}$ database evaluation grades (Table 7) have only one area with a lower grade (question 14 in Table 2). The first scenario evaluation grades (Table 3 ) have several questions with lower grades, which are grouped into one excerpt for an easier overview (Table 15). The company D's evaluation grades (Table 5) present a huge number of questions with lower grades and that excerpt is much larger (Table 16). 
Table 12.

Resemblance analysis of the evaluation grades for the first quality evaluation ( \%).

\begin{tabular}{|c|c|c|c|c|c|c|c|c|c|c|c|c|c|c|c|c|}
\hline & A-1 & A-2 & A-3 & B-1 & B-2 & B-3 & B-4 & B-5 & C-1 & C-2 & C-3 & C-4 & C-5 & C-6 & C-7 & C-8 \\
\hline A-1 & 100 & 80.83 & 92.50 & 92.50 & 91.67 & 93.33 & 92.50 & 92.50 & 87.50 & 87.50 & 85.83 & 81.67 & 88.33 & 87.50 & 76.67 & 90.00 \\
\hline A-2 & 80.83 & 100 & 88.33 & 73.33 & 74.17 & 74.17 & 73.33 & 75.00 & 71.67 & 73.33 & 73.33 & 79.17 & 72.50 & 71.67 & 79.17 & 74.17 \\
\hline A-3 & 2.50 & 88.33 & 100 & 5.00 & 4.17 & 85.83 & 85.00 & 85.00 & 83.33 & 85.00 & 81.67 & 84.17 & 84.17 & 3.33 & 0.83 & 85.83 \\
\hline$D-1$ & 2.50 & 73.33 & 85.00 & 100 & 99.17 & 99.17 & 100 & 98.33 & 85.00 & 85.00 & 83.33 & 77.50 & 85.83 & 85.00 & 74.17 & 85.83 \\
\hline B-2 & 91.67 & 74.17 & 84.17 & 99.17 & 100 & 98.33 & 99.17 & 99.17 & 84.17 & 84.17 & 84.17 & 76.67 & 85.00 & 84.17 & 75.00 & 85.00 \\
\hline B-3 & 3.33 & 74.17 & 85.83 & 9.17 & 98.33 & 100 & 99.17 & 99.17 & 84.17 & 84.17 & 84.17 & 78.33 & 85.00 & 84.17 & 73.33 & 86.67 \\
\hline B-4 & 9) 50 & 73.33 & 5.00 & 100 & 99.17 & 99.17 & 100 & 98.33 & 85.00 & 85.00 & 83.33 & 77.50 & 85.83 & 85.00 & 74.17 & 85.83 \\
\hline B-5 & 92.50 & 75.00 & 85.00 & 98.33 & 99.17 & 99.17 & 98.33 & 100 & 83.33 & 83.33 & 85.00 & 77.50 & 84.17 & 83.33 & 74.17 & 85.83 \\
\hline $2-1$ & 87.50 & 71.67 & 83.33 & 85.00 & 84.17 & 84.17 & 85.00 & 83.33 & 100 & 98.33 & 96.67 & 90.83 & 99.17 & 100 & 89.17 & 97.50 \\
\hline C-2 & 87.50 & 73.33 & 85.00 & 85.00 & 84.17 & 84.17 & 85.00 & 83.33 & 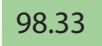 & 100 & 95.00 & 89.17 & 99.17 & 98.33 & 87.50 & 97.50 \\
\hline C-3 & 85.83 & 73.33 & 81.67 & 83.33 & 84.17 & 84.17 & 83.33 & 85.00 & 96.67 & 95.00 & 100 & 90.83 & 95.83 & 96.67 & 89.17 & 95.83 \\
\hline C-4 & 81.67 & 79.17 & 84.17 & 77.50 & 76.67 & 78.33 & 77.50 & 77.50 & 90.83 & 89.17 & 90.83 & 100 & 90.00 & 90.83 & 95.00 & 91.67 \\
\hline C & 88.33 & 72.5 & $T .1$ & 85.83 & 85.00 & 8 & 8 & 8 & 99.17 & 7 & 9 & 9 & 1 & 7 & 3 & 9 \\
\hline C-6 & 87.50 & 71.67 & 83.33 & 85.00 & 84.17 & 84.17 & 85.00 & 83.33 & 100 & 98.33 & 96.67 & 90.83 & 99.17 & 100 & 89.17 & 97.50 \\
\hline C-7 & 76.67 & 79.17 & 80.83 & 74.17 & 75.00 & 73.33 & 74.17 & 74.17 & 89.17 & 87.50 & 89.17 & 95.00 & 88.33 & 89.17 & 100 & 86.67 \\
\hline C-8 & 90.00 & 74.17 & 85.83 & 85.83 & 85.00 & 86.67 & 85.83 & 85.83 & 97.50 & 97.50 & 95.83 & 91.67 & 98.33 & 97.50 & 86.67 & 100 \\
\hline
\end{tabular}

Table 13.

Resemblance analysis of the evaluation grades for company D (\%).

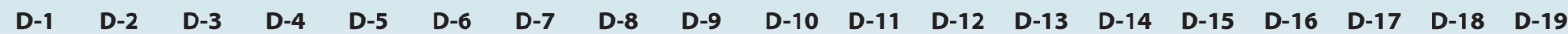
$\begin{array}{llllllllllllllllllll}\text { D-1 } & 100 & 72.50 & 66.67 & 81.67 & 79.17 & 70.00 & 64.17 & 72.50 & 90.83 & 65.83 & 75.00 & 74.17 & 68.33 & 68.33 & 72.50 & 95.00 & 75.83 & 67.50 & 80.00\end{array}$ $\begin{array}{lllllllllllllllllllll}\text { D-2 } & 72.50 & 100 & 89.17 & 90.83 & 88.33 & 94.17 & 83.33 & 91.67 & 71.67 & 85.00 & 94.17 & 93.33 & 90.83 & 89.17 & 96.67 & 74.17 & 93.33 & 83.33 & 87.50\end{array}$ $\begin{array}{llllllllllllllllllllllll}\text { D-3 } & 66.67 & 89.17 & 100 & 83.33 & 82.50 & 86.67 & 89.17 & 90.83 & 70.83 & 92.50 & 85.00 & 84.17 & 90.00 & 93.33 & 87.50 & 70.00 & 85.83 & 90.83 & 86.67\end{array}$ $\begin{array}{llllllllllllllllllllll}\text { D-4 } & 81.67 & 90.83 & 83.33 & 100 & 85.83 & 86.67 & 79.17 & 87.50 & 77.50 & 80.83 & 91.67 & 87.50 & 85.00 & 85.00 & 90.83 & 81.67 & 92.50 & 80.83 & 80.83\end{array}$ $\begin{array}{llllllllllllllllllll}\text { D-5 } & 79.17 & 88.33 & 82.50 & 85.83 & 100 & 84.17 & 75.00 & 86.67 & 78.33 & 78.33 & 84.17 & 86.67 & 82.50 & 82.50 & 86.67 & 80.83 & 91.67 & 80.00 & 90.83\end{array}$ \begin{tabular}{llllllllllllllllllll} 
D-6 & 70.00 & 94.17 & 86.67 & 86.67 & 84.17 & 100 & 89.17 & 87.50 & 70.83 & 85.83 & 90.00 & 94.17 & 93.33 & 90.00 & 95.83 & 71.67 & 89.17 & 82.50 & 83.33 \\
\hline
\end{tabular} $\begin{array}{lllllllllllllllllllllll}\text { D-7 } & 64.17 & 83.33 & 89.17 & 79.17 & 75.00 & 89.17 & 100 & 81.67 & 70.00 & 93.33 & 82.50 & 83.33 & 84.17 & 90.83 & 85.00 & 67.50 & 78.33 & 91.67 & 80.83\end{array}$ $\begin{array}{lllllllllllllllllllll}\text { D-8 } & 72.50 & 91.67 & 90.83 & 87.50 & 86.67 & 87.50 & 81.67 & 100 & 73.33 & 88.33 & 90.83 & 88.33 & 89.17 & 90.83 & 91.67 & 74.17 & 91.67 & 83.33 & 89.17\end{array}$ $\begin{array}{lllllllllllllllllllllll}\text { D-9 } & 90.83 & 71.67 & 70.83 & 77.50 & 78.33 & 70.83 & 70.00 & 73.33 & 100 & 70.00 & 72.50 & 73.33 & 70.83 & 72.50 & 71.67 & 95.83 & 73.33 & 70.00 & 82.50\end{array}$

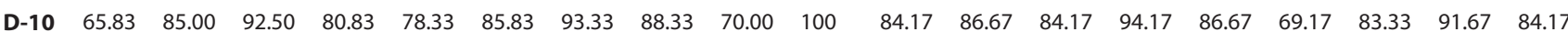
$\begin{array}{lllllllllllllllllllll}\text { D-11 } & 75.00 & 94.17 & 85.00 & 91.67 & 84.17 & 90.00 & 82.50 & 90.83 & 72.50 & 84.17 & 100 & 92.50 & 90.00 & 88.33 & 92.50 & 76.67 & 90.83 & 82.50 & 86.67\end{array}$ $\begin{array}{llllllllllllllllllll}\text { D-12 } & 74.17 & 93.33 & 84.17 & 87.50 & 86.67 & 94.17 & 83.33 & 88.33 & 73.33 & 86.67 & 92.50 & 100 & 87.50 & 90.83 & 95.00 & 75.83 & 90.00 & 83.33 & 87.50\end{array}$ $\begin{array}{llllllllllllllllllll}\text { D-13 } & 68.33 & 90.83 & 90.00 & 85.00 & 82.50 & 93.33 & 84.17 & 89.17 & 70.83 & 84.17 & 90.00 & 87.50 & 100 & 86.67 & 90.83 & 71.67 & 87.50 & 82.50 & 83.33\end{array}$ $\begin{array}{llllllllllllllllllllll}\text { D-14 } & 68.33 & 89.17 & 93.33 & 85.00 & 82.50 & 90.00 & 90.83 & 90.83 & 72.50 & 94.17 & 88.33 & 90.83 & 86.67 & 100 & 92.50 & 71.67 & 87.50 & 89.17 & 88.33\end{array}$ $\begin{array}{llllllllllllllllllllll}\text { D-15 } & 72.50 & 96.67 & 87.50 & 90.83 & 86.67 & 95.83 & 85.00 & 91.67 & 71.67 & 86.67 & 92.50 & 95.00 & 90.83 & 92.50 & 100 & 74.17 & 93.33 & 83.33 & 87.50\end{array}$ $\begin{array}{lllllllllllllllllllllll}\text { D-16 } & 95.00 & 74.17 & 70.00 & 81.67 & 80.83 & 71.67 & 67.50 & 74.17 & 95.83 & 69.17 & 76.67 & 75.83 & 71.67 & 71.67 & 74.17 & 100 & 77.50 & 70.83 & 83.33\end{array}$ $\begin{array}{lllllllllllllllllllll}\text { D-17 } & 75.83 & 93.33 & 85.83 & 92.50 & 91.67 & 89.17 & 78.33 & 91.67 & 73.33 & 83.33 & 90.83 & 90.00 & 87.50 & 87.50 & 93.33 & 77.50 & 100 & 81.67 & 87.50\end{array}$ $\begin{array}{llllllllllllllllllllll}\text { D-18 } & 67.50 & 83.33 & 90.83 & 80.83 & 80.00 & 82.50 & 91.67 & 83.33 & 70.00 & 91.67 & 82.50 & 83.33 & 82.50 & 89.17 & 83.33 & 70.83 & 81.67 & 100 & 87.50\end{array}$ $\begin{array}{llllllllllllllllllll}\text { D-19 } & 80.00 & 87.50 & 86.67 & 80.83 & 90.83 & 83.33 & 80.83 & 89.17 & 82.50 & 84.17 & 86.67 & 87.50 & 83.33 & 88.33 & 87.50 & 83.33 & 87.50 & 87.50 & 100\end{array}$ 
Table 14.

Resemblance analysis of the evaluation grades for company $\mathrm{E}(\%)$

\begin{tabular}{llllllllll} 
& E-1 & E-2 & E-3 & E-4 & E-5 & E-6 & E-7 & E-8 & E-9 \\
\hline E-1 & 100 & 99.17 & 99.17 & 98.33 & 99.17 & 99.17 & 99.17 & 99.17 & 99.17 \\
\hline E-2 & 99.17 & 100 & 100 & 99.17 & 98.33 & 100 & 100 & 98.33 & 98.33 \\
\hline E-3 & 99.17 & 100 & 100 & 99.17 & 98.33 & 100 & 100 & 98.33 & 98.33 \\
\hline E-4 & 98.33 & 99.17 & 99.17 & 100 & 99.17 & 99.17 & 99.17 & 99.17 & 99.17 \\
\hline E-5 & 99.17 & 98.33 & 98.33 & 99.17 & 100 & 98.33 & 98.33 & 100 & 100 \\
\hline E-6 & 99.17 & 100 & 100 & 99.17 & 98.33 & 100 & 100 & 98.33 & 98.33 \\
\hline E-7 & 99.17 & 100 & 100 & 99.17 & 98.33 & 100 & 100 & 98.33 & 98.33 \\
\hline E-8 & 99.17 & 98.33 & 98.33 & 99.17 & 100 & 98.33 & 98.33 & 100 & 100 \\
\hline E-9 & 99.17 & 98.33 & 98.33 & 99.17 & 100 & 98.33 & 98.33 & 100 & 100 \\
\hline
\end{tabular}

Table 15.

Questions for the first quality evaluation with lower evaluation grades.

\begin{tabular}{|c|c|c|c|c|c|c|c|c|c|c|c|c|c|c|c|c|}
\hline \multirow{2}{*}{ Question } & \multicolumn{16}{|c|}{ Database } \\
\hline & A-1 & A-2 & $A-3$ & B-1 & B-2 & B-3 & B-4 & B-5 & C-1 & $C-2$ & $C-3$ & $C-4$ & $C-5$ & C-6 & C-7 & C-8 \\
\hline 01. & 5 & 3 & 5 & 5 & 4 & 5 & 5 & 4 & 5 & 5 & 4 & 5 & 5 & 5 & 4 & 5 \\
\hline 02. & 4 & 3 & 4 & 5 & 5 & 5 & 5 & 5 & 4 & 4 & 5 & 4 & 4 & 4 & 4 & 4 \\
\hline 06. & 4 & 3 & 4 & 4 & 4 & 4 & 4 & 4 & 4 & 4 & 3 & 3 & 4 & 4 & 3 & 4 \\
\hline 08. & 5 & 3 & 4 & 5 & 5 & 5 & 5 & 5 & 5 & 4 & 5 & 5 & 5 & 5 & 5 & 5 \\
\hline 10. & 4 & 4 & 4 & 5 & 5 & 5 & 5 & 5 & 4 & 4 & 4 & 4 & 4 & 4 & 4 & 4 \\
\hline 11. & 5 & 5 & 5 & 4 & 4 & 4 & 4 & 4 & 2 & 3 & 2 & 2 & 3 & 2 & 1 & 3 \\
\hline 12. & 4 & 4 & 4 & 5 & 5 & 5 & 5 & 5 & 3 & 3 & 3 & 3 & 3 & 3 & 3 & 3 \\
\hline 14. & 4 & 4 & 4 & 5 & 5 & 5 & 5 & 5 & 4 & 4 & 4 & 4 & 4 & 4 & 4 & 4 \\
\hline 15. & 5 & 1 & 3 & 5 & 5 & 5 & 5 & 5 & 5 & 5 & 5 & 1 & 5 & 5 & 1 & 5 \\
\hline 16. & 5 & 1 & 2 & 5 & 5 & 5 & 5 & 5 & 5 & 5 & 5 & 3 & 5 & 5 & 1 & 5 \\
\hline 20. & 4 & 4 & 4 & 3 & 3 & 3 & 3 & 3 & 4 & 4 & 4 & 4 & 4 & 4 & 4 & 4 \\
\hline 21. & 5 & 5 & 5 & 5 & 5 & 5 & 5 & 5 & 4 & 4 & 4 & 4 & 4 & 4 & 4 & 4 \\
\hline 23. & 5 & 2 & 4 & 5 & 5 & 5 & 5 & 5 & 4 & 4 & 4 & 3 & 4 & 4 & 3 & 4 \\
\hline 25. & 5 & 2 & 4 & 5 & 5 & 5 & 5 & 5 & 4 & 4 & 4 & 4 & 4 & 4 & 4 & 4 \\
\hline 26. & 4 & 4 & 4 & 3 & 3 & 3 & 3 & 3 & 5 & 5 & 5 & 5 & 5 & 5 & 5 & 5 \\
\hline 27. & 5 & 2 & 4 & 5 & 5 & 5 & 5 & 5 & 5 & 5 & 5 & 5 & 5 & 5 & 5 & 5 \\
\hline 30. & 5 & 5 & 5 & 5 & 5 & 5 & 5 & 5 & 4 & 4 & 4 & 3 & 4 & 4 & 3 & 4 \\
\hline
\end{tabular}


Table 16.

Company D's questions with lower evaluation grades.

\begin{tabular}{|c|c|c|c|c|c|c|c|c|c|c|c|c|c|c|c|c|c|c|c|}
\hline \multirow{2}{*}{ Question } & \multicolumn{19}{|c|}{ Database } \\
\hline & D-1 & D-2 & D-3 & D-4 & D-5 & D-6 & D-7 & D-8 & D-9 & D-10 & D-11 & D-12 & D-13 & D-14 & D-15 & D-16 & D-17 & D-18 & D-19 \\
\hline 01. & 3 & 4 & 4 & 4 & 4 & 5 & 5 & 4 & 3 & 4 & 4 & 4 & 5 & 4 & 4 & 3 & 4 & 4 & 4 \\
\hline 02. & 3 & 5 & 5 & 5 & 4 & 5 & 5 & 4 & 3 & 5 & 5 & 5 & 5 & 5 & 5 & 3 & 4 & 5 & 5 \\
\hline 03. & 2 & 4 & 4 & 3 & 4 & 5 & 5 & 4 & 5 & 5 & 4 & 5 & 5 & 5 & 5 & 4 & 4 & 4 & 4 \\
\hline 04. & 5 & 4 & 4 & 4 & 2 & 5 & 5 & 4 & 5 & 5 & 4 & 5 & 4 & 4 & 4 & 5 & 2 & 4 & 4 \\
\hline 06. & 2 & 4 & 4 & 3 & 4 & 5 & 5 & 4 & 3 & 4 & 4 & 5 & 4 & 5 & 5 & 3 & 4 & 4 & 4 \\
\hline 08. & 3 & 5 & 4 & 4 & 3 & 5 & 5 & 5 & 2 & 5 & 5 & 5 & 4 & 5 & 5 & 2 & 4 & 4 & 3 \\
\hline 09. & 3 & 5 & 4 & 4 & 4 & 4 & 4 & 4 & 2 & 4 & 5 & 5 & 4 & 4 & 4 & 3 & 4 & 4 & 4 \\
\hline 10. & 1 & 2 & 2 & 2 & 2 & 2 & 2 & 2 & 1 & 2 & 1 & 2 & 1 & 2 & 2 & 1 & 2 & 2 & 2 \\
\hline 11. & 1 & 1 & 1 & 1 & 1 & 1 & 1 & 1 & 1 & 1 & 1 & 1 & 1 & 1 & 1 & 1 & 1 & 1 & 1 \\
\hline 12. & 1 & 1 & 1 & 1 & 1 & 1 & 1 & 1 & 1 & 1 & 1 & 1 & 1 & 1 & 1 & 1 & 1 & 1 & 1 \\
\hline 13. & 1 & 1 & 1 & 1 & 1 & 1 & 4 & 1 & 1 & 4 & 1 & 1 & 1 & 1 & 1 & 1 & 1 & 4 & 1 \\
\hline 14. & 1 & 1 & 4 & 1 & 1 & 1 & 4 & 2 & 2 & 4 & 1 & 1 & 1 & 3 & 1 & 1 & 1 & 4 & 2 \\
\hline 15. & 2 & 1 & 4 & 1 & 2 & 1 & 4 & 3 & 3 & 4 & 1 & 1 & 1 & 4 & 1 & 2 & 1 & 5 & 3 \\
\hline 16. & 1 & 1 & 3 & 1 & 1 & 1 & 3 & 1 & 2 & 3 & 1 & 1 & 2 & 3 & 1 & 2 & 1 & 3 & 2 \\
\hline 17. & 1 & 1 & 1 & 1 & 1 & 1 & 1 & 1 & 1 & 1 & 1 & 1 & 1 & 1 & 1 & 1 & 1 & 1 & 1 \\
\hline 18. & 1 & 1 & 1 & 1 & 1 & 1 & 1 & 1 & 1 & 1 & 1 & 1 & 1 & 1 & 1 & 1 & 1 & 1 & 1 \\
\hline 20. & 2 & 5 & 5 & 3 & 4 & 5 & 5 & 4 & 2 & 4 & 4 & 4 & 5 & 4 & 5 & 2 & 3 & 5 & 4 \\
\hline 21. & 2 & 4 & 4 & 3 & 4 & 5 & 5 & 4 & 3 & 4 & 4 & 4 & 5 & 4 & 4 & 3 & 4 & 5 & 4 \\
\hline 23. & 1 & 4 & 5 & 2 & 4 & 4 & 2 & 5 & 1 & 4 & 2 & 4 & 5 & 4 & 4 & 1 & 4 & 3 & 3 \\
\hline 24. & 1 & 4 & 5 & 4 & 4 & 4 & 4 & 5 & 1 & 5 & 4 & 4 & 5 & 4 & 4 & 1 & 4 & 4 & 3 \\
\hline 25. & 1 & 4 & 4 & 3 & 3 & 4 & 4 & 5 & 1 & 4 & 5 & 4 & 5 & 4 & 4 & 1 & 4 & 4 & 4 \\
\hline 26. & 1 & 1 & 1 & 1 & 1 & 1 & 1 & 1 & 1 & 1 & 1 & 1 & 1 & 1 & 1 & 1 & 1 & 1 & 1 \\
\hline 28. & 5 & 4 & 4 & 5 & 3 & 4 & 4 & 5 & 4 & 5 & 5 & 5 & 4 & 5 & 5 & 5 & 5 & 5 & 5 \\
\hline 30. & 1 & 5 & 5 & 5 & 1 & 5 & 5 & 5 & 1 & 5 & 5 & 3 & 5 & 5 & 5 & 1 & 5 & 3 & 1 \\
\hline
\end{tabular}

\section{DISCUSSION}

As all the first scenario databases are made by same development team, it was expected that the evaluation would present very similar results to the results obtained during testing of evaluation methodology for ship's Planned Maintenance System database. The expected results should be equalized to the summation of grades, i.e. average grade value, quite big resemblance of the grades, and a similar list of questions with grades 1,2 , and 3 .

The analysis of company A's databases showed unevenness in the quality of the data as well as in the deficiencies. The average grade value ranges from 3.967 to 4.733 , and the resemblance of grades varies from 80 to $92 \%$. As there are only three databases in the company, the sample is too small to define any reliable finding (Tversky and Kahneman, 1971).

The analysis of company B's database evaluation results shows a remarkable overall uniformity, in the average grade, resemblance (Figure 3), and deficiencies. The average grade difference is very small, varying from 4.733 to 4.800 , resemblance from $98.33 \%$ to $100 \%$, major deficiencies are noted in questions 20 and 26 with all company databases. 


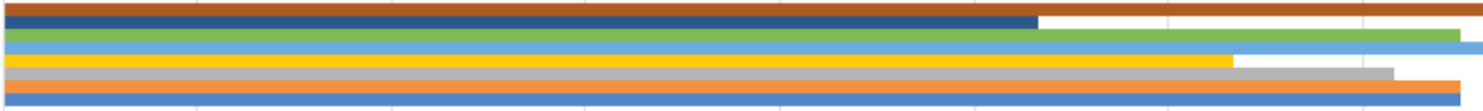

C-7

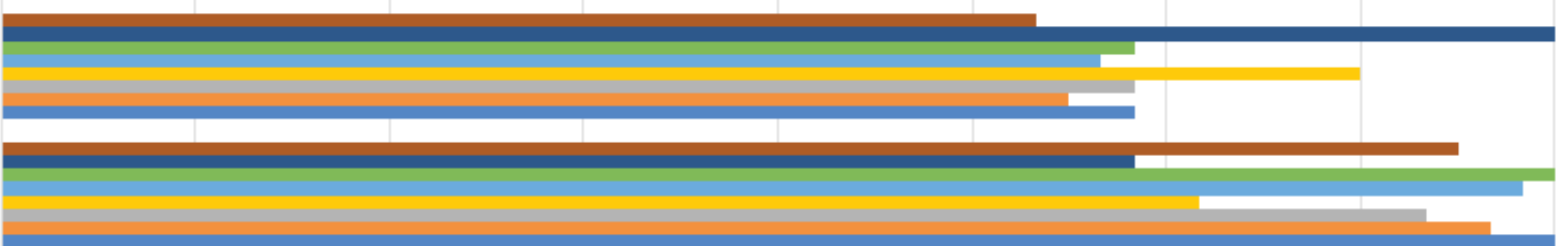

C- 6

C-5

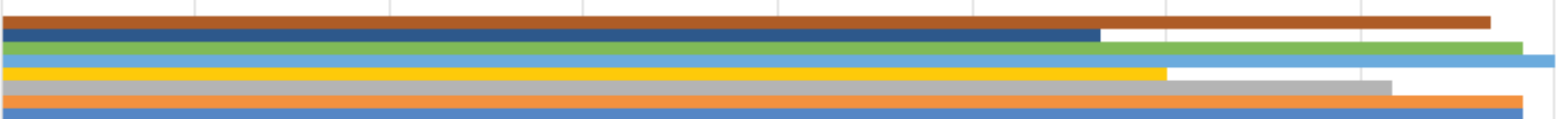

C-4

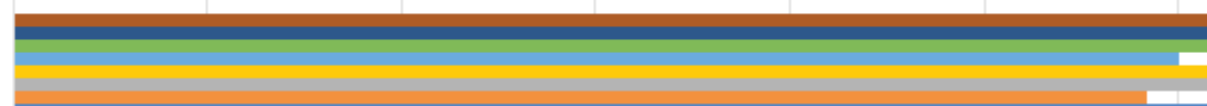

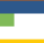

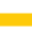

C-3

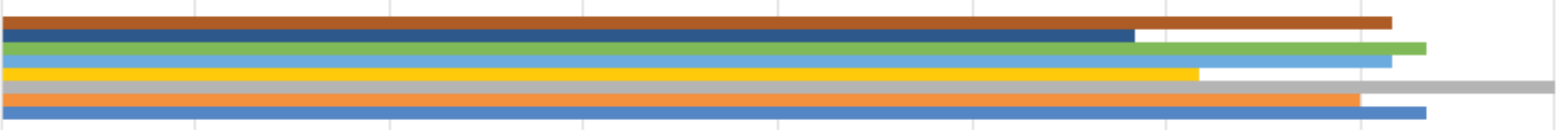

C-2

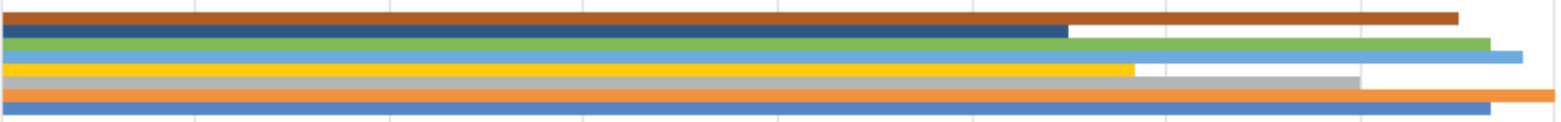

C-1

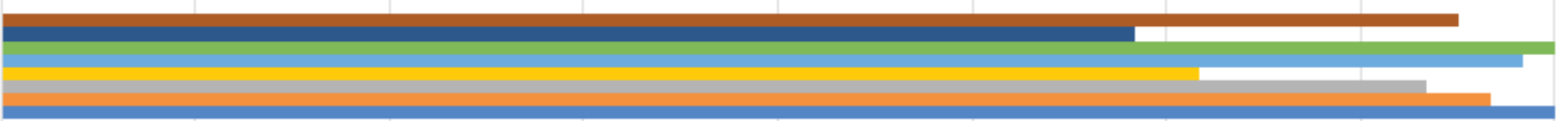

60

65

70

75

80

85

90

95

C-8 $\square \mathrm{C}-7 \square \mathrm{C}-6 \square \mathrm{C}-5 \square \mathrm{C}-4 \square \mathrm{C}-3 \square \mathrm{C}-2 \square \mathrm{C}-1$

Figure 4.

Company C's resemblance of grades (in \%).

Remark: The colour bar (The colour for each database is shown in the small squares below the diagram) indicates the resemblance of the databases compared, with the databases indicated on the ordinate.

The deviations of results, i.e. the deficiencies noted during the evaluation of databases A-2, C-4 and C-7, are connected to the lack of ship's instruction books and documents during the database development. The lack of documents is quite a common case when the database is developed for the second-hand vessels where some instruction books and technical documentation are often missing.

The comparison of the evaluation grades of the databases tested according to the first scenario shows resemblance of grade (and equivalence of the average grade) drops when compared with the databases of different companies (Figure 5).
The comparison shows that the databases developed by same team will have significant overall resemblance, which varies between $80 \%$ and $90 \%$ (Table 12 and Figure 5), when developed for different shipowners. The resemblance increases to over 90 $\%$ when the databases are developed for the same shipowner. This is emphasized in case of company B, where the grades for some databases are the same (Figure 5). The analysis of major deficiencies shows that the deficiencies differ from company to company. All this points to an existing control on the shipowner's side (shipping companies) and to a uniformity of quality within each company. 


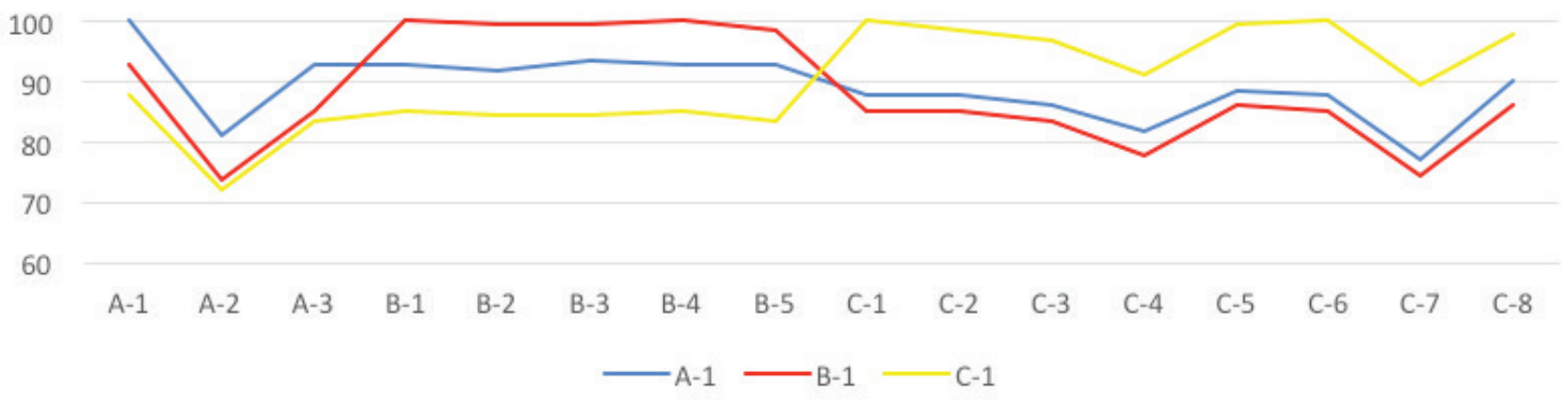

Figure 5.

Comparison of the first scenario database evaluation grades resemblance (in \%).

Comment: One database per company is shown for a better overview.

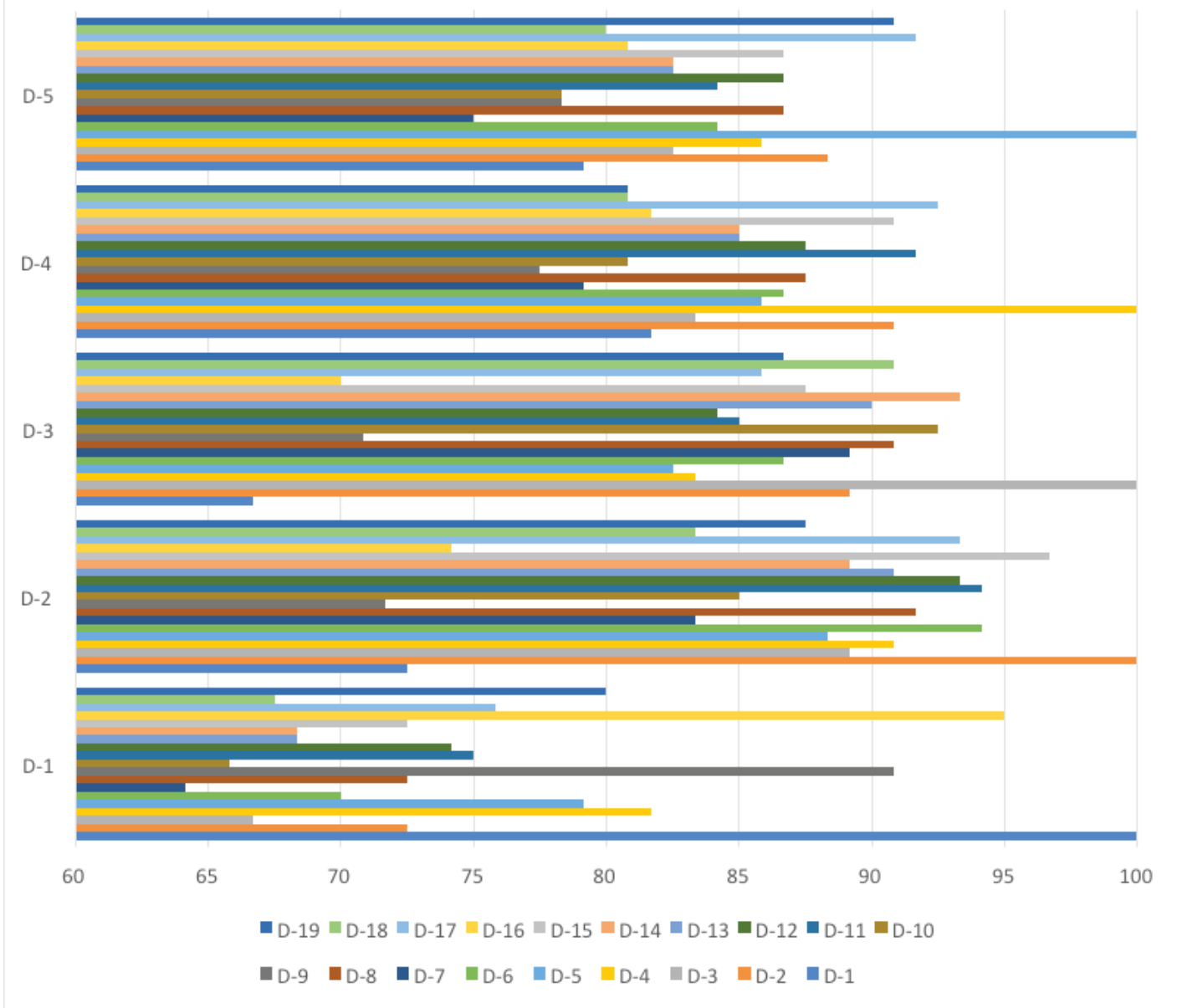

Figure 6.

Company D resemblance of grades (in \%).

Comment: Due to size issues, comparison of the first five databases is given in the diagram.

Remark: The colour bar (The colour for each database is shown in the small squares below the diagram) indicates the resemblance of the databases compared, with the databases indicated on the ordinate. 
The analysis of the evaluation grade results for the second scenario shows two completely different findings. The company $D$ evaluation grades show a bad condition in general, which is obviously represented by a bad average grade (from 2,467 to $3,833)$, a larger number of noted deficiencies and big differences of resemblance analysis - from 64 to $95 \%$ (Figure 6), as well as the differences of questions with noted deficiencies. The databases of this company are made by several development teams, each having their own rules, mistakes, criteria, etc. Big differences of evaluation grades signify that during the development of the databases, a corrective mechanism for equalizing quality did not exist. Some databases, like D-7 and D-10, have a relatively good average grade and with little effort would be acceptable. At the same time, D-1 and D-16 need a huge effort to improve their quality to an acceptable level.

The resemblance of the grades across the company $\mathrm{E}$ (Figure 7) is very high (from 98.33 to $100 \%$ ) as well as the uniformity of the average grades (from 4.533 to 4.600 ). The conformity of all the results continues with the questions with lower grades, the only noted question is 14 . As this company also develops their databases by several development teams, obtaining results like this is possible only if all the teams have clearly marked guidelines and a well-organized and performed database checking.

E-9

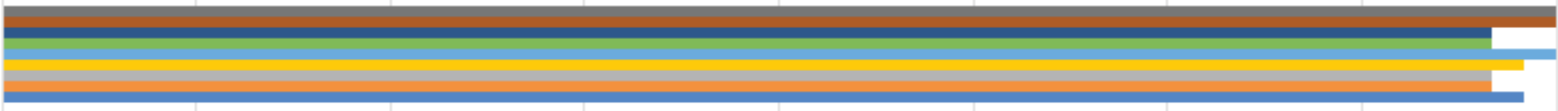

E-8

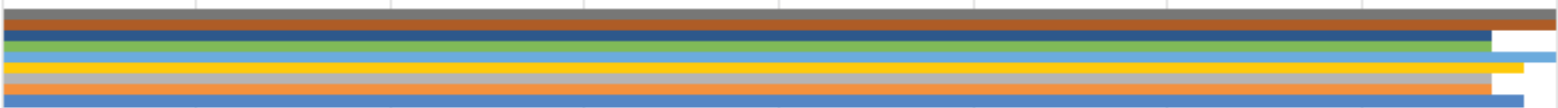

E-7

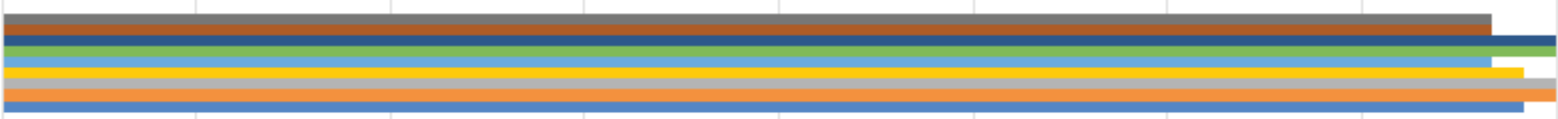

E-6

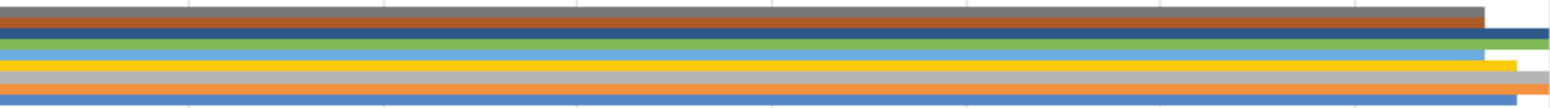

E-5

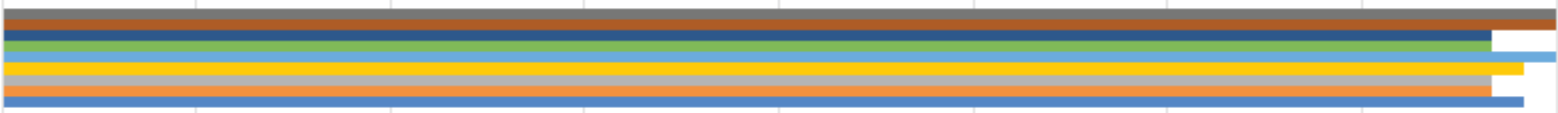

$E-4$

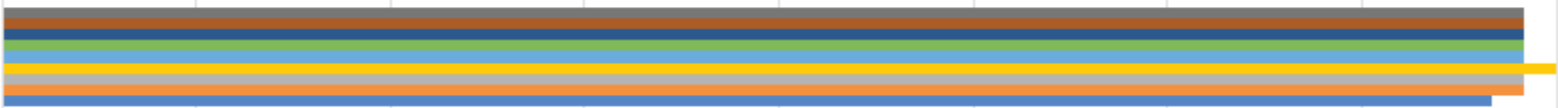

E-3

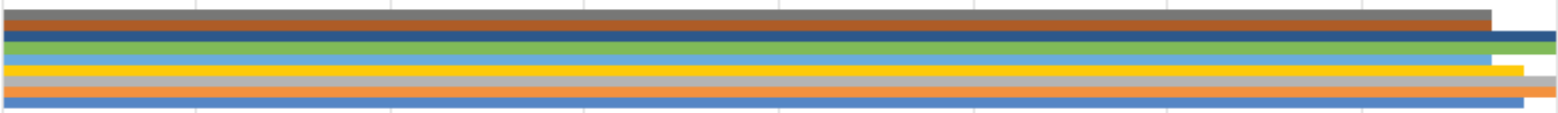

E-2

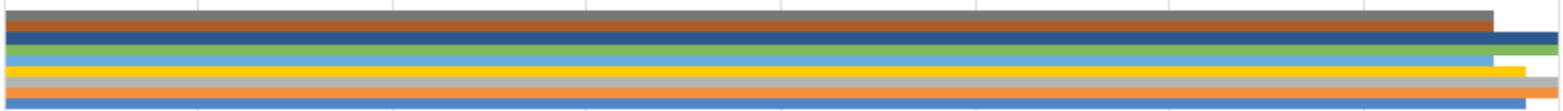

E-1

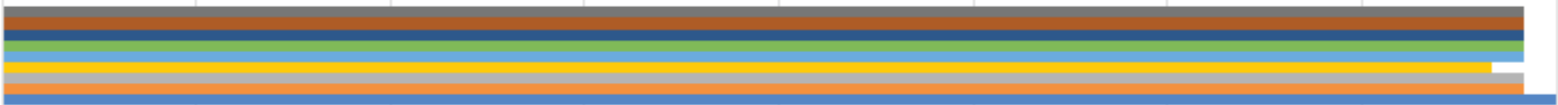

60

65

70

75

80

85

90

95

100

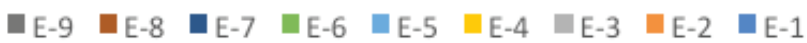

Figure 7.

Company E's resemblance of grades (in \%).

Remark: The colour bar (The colour for each database is shown in the small squares below the diagram.) indicates the resemblance of the databases compared, with the databases indicated on the ordinate. 
Time used for administration and correction of database should be considered with certain dose of reserve because it depends upon shipboard user access rights policy and what shipboard user can perform in the system. If the shipboard user has restrictive user rights, the office user will work more and vice versa. This time vary from 0.3 hours per day in the company A to 1.5 hours per day in the company B. An exception is company $D$ where that time is negligible, and it is not enough for even a basic oversight of performed activities in the system, i.e. supervision of shipboard users. Adding this information to very bad condition of company D databases, it becomes obvious that there is neglect of computerized Planned Maintenance System, i.e. neglect of shipowner's role during database development process.

The company E's results showed that there was a high-quality supervision and check of the data entered, which increased the quality of the data in the database and, consequently, increased efficiency and reliability, reduced errors and user training, increased system and information acceptance (Bias and Mayhew, 1994) (Karat et. al., 1992).

\section{CONCLUSION}

Database ordering and development sequence diagram show mutual dependence of the shipowner and the developer during the whole process. It also describes the points and shipowner's actions which directly influence the quality of data in computerized planned maintenance database. The research shows in detail how the shipowner affects database quality grades and how this influence can be numerically expressed.

The first scenario evaluated the databases built by one development team for three different shipowners. The research shows that database quality grades have an overall resemblance from 80 to $100 \%$. If the resemblance of grades is compared for different shipowners, it varies from $80 \%$ to $90 \%$, and with one shipowner it goes from $90 \%$ to $100 \%$. The impact of the shipowner towards quality evaluation grades, according to findings, can be measured as increase of resemblance of grades by $10 \%$.

The evaluation of databases performed according to the second scenario, in which several teams build databases for companies, results in two conclusions. The company D's resemblance analysis results show that the evaluation grades of a single company will vary significantly (from $64 \%$ to 95 $\%$ ) if the shipowner's impact is missing (or it is removed). The databases will have uneven quality and usability. In contrast to this, company E's resemblance analysis (from 98.33 to $100 \%$ ) shows that the grades will be very uniform if there is the control and supervision by the shipowner. In that case, the databases will have very similar quality and usability.

All the conclusions of research according to the first scenario can be applied to the results obtained during the testing of evaluation methodology for ship's Planned Maintenance System database (Table 1), where the similarity of results can be noted. The resemblance of database evaluation grades within one company (databases 1 to 4 ) is between $95.00 \%$ and 99.17 $\%$, which is in accordance with the first scenario results. The resemblance of grades decreases to the range from 86.66 to $90.83 \%$ when compared with a database developed for another shipowner (database 5), also in accordance with the first scenario results. The comparison provides identical results, which solves the problem of unexpected results of a small sample.

It has been found out that Ship's Planned Maintenance System database evaluation grades will have a great resemblance within one company (higher than $90 \%$, often over $95 \%$ ), if there is good shipowner's supervision of the database development process and a well organized and carried-out acceptance test. This fact is valid for all the databases of one shipowner, whereas the number of development teams has no relevance.

\section{REFERENCES}

ABS rules for conditions of classification, part 1, (2016), available at: https://ww2. eagle.org/content/dam/eagle/rules-and-guides/current/generic/Generic_Rules_ Conditions_Classification_Material_Welding_Survey_2016/Part_1_Ships_e-Aug16. pdf

Basili, V. R., Caldiera, G. and Rombach, H. D., (1994), Goal Question Metric (GQM) approach, Encyclopedia of software engineering 1: pp. 528-532., available at: https://doi.org/10.1002/0471028959.sof142

Beynon-Davies, P., (2004), Database Systems. Houndmills, Basingstoke, UK: Palgrave, pp. 195-208., available at: https://pdfs.semanticscholar.org/438f/ f618ba74abdb670cf6fd5eff15ebcf496300.pdf, [accessed 14 December 2017].

Bias, R. G., Mayhew, D. J., (1994), Cost-Justifying Usability. Academic Press, Boston, available at:

https://doi.org/10.1016/b978-0-12-095811-5.x5000-7

CRS rules for classification of ships, Part 1, Chapter 1, (2014), available at: http:// www.crs.hr/Portals/0/docs/Pravila/klasifikacijska/Rules \%20for \%20the \%20 classification \%20of \%20ships, \%20Pt. \%201, \%20Ch.1, \%202014.pdf, [accessed 26 November 2017].

Davis, G.B., (1982), Strategies for information requirements determination, IBM Systems Journal 21(1), pp. 4 - 30., available at:

https://doi.org/10.1147/sj.211.0004

Davis, G.B., Olson, M.H., (1985), Management Information Systems: Conceptual Foundations, Structure and Development, 2nd edition, New York: McGraw-Hill, pp. 447-500.

DNV rules for classification of ships, Part 7, Chapter 8, (2003), available at: https:// rules.dnvgl.com/docs/pdf/DNV/rulesship/2004-01/ts708.pdf, [accessed 20 November 2017].

Gackowski, Z. J., (2006), Redefining Information Quality and its Measuring: The Operations Management Approach, Proceedings of the 11th International Conference on Information Quality (ICIQ), pp. 399-419.,

Guidelines for flag State inspections under the Maritime Labour Convention, (2006), available at: http://www.ics-shipping.org/docs/default-source/ILO-MLC-docs/iloguidelines-for-flag-state-inspections-under-the-mlc.pdf?sfvrsn=4, [accessed 26 November 2017]. 
Karat, C. M., Campbell, R. and Fiegel, T., (1992), Comparison of empirical testing and walkthrough methods in user interface evaluation, Proceedings of SIGCHI'92, Monterey, California, US, May 3-7, pp. 397-404., available at: https://doi.org/10.1145/142750.142873

Manuel, M. E., (2012), Maritime Risk and Organizational Learning, Ashgate Publishing, pp. 17.

Maydanchik, A., (2007), Data Quality Assessment. Bradley Beach, NJ: Technics Publications, pp. 11.

Miller, R., Collins, C., (2001), Acceptance testing. Proc. XP / Agile Universe, available at: $\quad$ http://www.dsc.ufcg.edu.br/ jacques/cursos/map/recursos/Testing05.pdf, [accessed 29 November 2017].

Rabin, M., (2002), Inference by believers in the law of small numbers. Quarterly Journal of Economics 117, pp. 775-816., available at: https://doi.org/10.1162/003355302760193896

Rogers, O., (2004), Acceptance Testing vs Unit Testing: A Developer's Perspective. Proc. XP / Agile Universe, Lecture Notes in Computer Science, Springer Verlag, Vol. 3134, pp. 22-31.
Stazić, L., Komar, I., Račić, N., (2017), Evaluation Methodology for Ship's Planned Maintenance System Database, Transactions on Maritime Science, 06(02), available at:

https://doi.org/10.7225/toms.v06.n02.002

Strong, D. M., Lee, Y. W. and Wang, R. Y., (1997), Communications of the ACM 40(5), available at: http://web.mit.edu/tdqm/www/winter/StrongLeeWangCACMMay97. pdf, [accessed 4 December 2017].

Tversky, A., Kahneman, D., (1971), Belief in the law of small numbers. Psychological bulletin 76(2), pp. 105-110., available at: https://doi.org/10.1037/h0031322

Wang, R. Y., Strong, D. M., (1996), Beyond Accuracy: What Data Quality Means to Data Consumers, Journal of Management Information Systems 12(4), pp. 5-33., available at:

https://doi.org/10.1080/07421222.1996.11518099

Watt, A., Eng, N., (2014). Database Design, 2nd edition, Bccampus, ch. 13, pp. 77. available at: http://solr.bccampus.ca:8001/bcc/file/5b6f010a-0563-44d4-94c567caa515d2c5/1/Database-Design-2nd-Edition-1481742440.pdf, [accessed 4 December 2017]. 